\title{
Fabricating Tubular Devices from Polymers of Lactic and Glycolic Acid for Tissue Engineering
}

\author{
D.J. MOONEY, Ph.D., ${ }^{1,2,3}$ C. BREUER, M.D., ${ }^{2}$ K. MCNAMARA, B.S., 1 \\ J.P. VACANTI, M.D., ${ }^{2}$ and R. LANGER, Sc.D. ${ }^{2}$
}

\begin{abstract}
Polymers of lactic and glycolic acid are attractive candidates to fabricate devides to transplant cells and engineer new tissues. These polymers are biocompatible, and exhibit a wide range of erosion times and mechanical properties. This manuscript describes the fabrication and characterization, in vitro and in vivo, of hollow, tubular devices from porous films of various polymers of this family. Porous films of these polymers were formed using a particulate leaching technique, and sealed around Teflon cylinders to form hollow tubular devices. The erosion rate of devices was controlled by the specific polymer utilized for fabrication, and ranged from months to years. Devices fabricated from a 50/50 copolymer of D,L-lactic acid and glycolic acid were completely eroded by 2 months, while devices fabricated from a homopolymer of L-lactic acid showed little mass loss after 1 year. Erosion times for devices fabricated from the other polymers [poly-(D,L-lactic acid) and a 85/15 copolymer] were between these two extremes. Devices were capable of resisting significant compressional forces $(150 \mathrm{mN})$ in vitro, and the compression resistance was controlled by the polymer utilized to fabricate the devices. The ability of the devices to maintain their structure after implantation into the mesentery or omentum of laboratory rats was also dependent of the specific polymer utilized to fabricate the device. These results indicate that it is possible to fabricate tubular devices for tissue engineering applications that exhibit a wide range of erosion rates and mechanical properties.
\end{abstract}

\section{INTRODUCTION}

$\mathbf{T}$ HE HUGE PERSONAL AND FINANCIAL COSTS associated with the loss or malfunction of tissues has spurred interest in engineering replacement tissues using cell transplantation. ${ }^{1}$ To engineer a new tissue from a group of originally disorganized cells it is first necessary to deliver the cells to a specific anatomic location. These cells, and any cells from the host tissue that are present, must then be provided with structural cues to organize appropriately. Our group has focused on fabricating polymer scaffolds to serve these pur-

\footnotetext{
${ }^{1}$ Department of Chemical Engineering, Massachusetts Institute of Technology, Cambridge, Massachusetts 02139.

${ }^{2}$ Department of Surgery, Harvard Medical School and the Children's Hospital, Boston, Massachusetts 02115.

${ }^{3}$ Current address: Departments of Biologic and Materials Sciences and Chemical Engineering, University of Michigan, Ann Arbor, Michigan 48109-2136.
} 
poses from biodegradable polymers. The degradation of the polymer after tissue development is complete can yield an entirely natural tissue. ${ }^{2}$ Homopolymers of lactic acid [poly-(L-lactic acid) (PLA) and poly-(D,Llactic acid) (PDLLA)], glycolic acid [polyglycolic acid (PGA)], and copolymers of lactic and glycolic acid (PLGA) are attractive candidates for fabricating tissue engineering scaffolds. These polymers have been used in medical devices for over 20 years, ${ }^{3}$ and are generally considered to be biocompatible. The crystallinity, mechanical properties, and erosion times of these polymers are regulated by the ratio of lactic:glycolic acid. ${ }^{4}$

There is considerable interest in engineering a variety of tubular tissue, including intestine, ${ }^{5}$ blood vessels, ${ }^{6}$ tracheas, ${ }^{7}$ and ureters. ${ }^{8}$ While the geometry of these tissues is similar, the necessary specifications (e.g., erosion time, mechanical properties) of devices used to engineer these different tissues will likely vary considerably. We have previously described a technique to fabricate hollow tubular structures from a 50/50 copolymer of lactic and glycolic acid (50/50 PLGA) that can be utilized to engineer tubular tissues. ${ }^{9}$ This report describes the fabrication and characterization of tubular cell delivery devices from a range of polymers of the lactic and glycolic acid. Devices with a wide range of compression resistance and erosion times can be fabricated by utilizing PLA, PDLLA, and PLGA.

\section{MATERIALS AND METHODS}

\section{Materials}

PLA, PDLLA, and the 85/15 and 50/50 PLGA were purchased from Medisorb (Cincinnati, OH), chloroform from Mallinckrodt (Paris, KY), polystyrene standards from Polysciences (Warrington, PA), aluminum backed tape from Cole-Parmer (Chicago, IL), phosphate-buffered saline and DMEM from Gibco (Grand Island, NY), Tmax film from Kodak, Lewis rats, 250 to $300 \mathrm{~g}$, from Charles River (Wilmington, MA), and methoxyflurane from Pitman-Moore Inc. (Mundelein, IL).

Molecular weights of the various polymers were determined by gel permeation chromatography (PerkinElmer, Series 10, Newton Centre, MA), using polystyrene standards to generate a calibration curve. PLA had a molecular weight $\left(M_{\mathrm{w}}\right)$ of $74,000\left(M_{\mathrm{w}} / M_{\mathrm{n}}=1.6\right)$; poly-(D,L-lactic) acid had $M_{\mathrm{w}}=77,000\left(M_{\mathrm{w}} / M_{\mathrm{n}}=\right.$ 1.8); $85 / 15$ PLGA had $M_{\mathrm{w}}=69,000\left(M_{\mathrm{w}} / M_{\mathrm{n}}=1.9\right) ; 50 / 50$ PLGA $M_{\mathrm{w}}=43,400\left(M_{\mathrm{w}} / M_{\mathrm{n}}=1.43\right)$. Differential scanning calorimetry was utilized to confirm the amorphous nature of all of the polymers except PLLA, which exhibited the expected crystallinity (not shown).

\section{Device Fabrication}

Hollow tubes were formed by a two-step process; porous films of the polymers were first fabricated, and these films were then formed into hollow tubes. To fabricate porous films, the polymer was dissolved in chloroform to form a $1.56 \%$ solution (w/v). Eight milliliters of this solution was cast into a 5 -cm glass petri dish covered with a sheet of aluminum backed tape. Sieved sodium chloride crystals $(150<d<250 \mu \mathrm{m})$ were dispersed evenly over the solution $(0.375 \mathrm{~g} \mathrm{NaCl} / \mathrm{dish})$, and the chloroform was allowed to evaporate at room temperature. A polymer film with entrapped $\mathrm{NaCl}$ particles resulted. The salt particles were leached out of the film by immersion in $800 \mathrm{ml}$ of deionized water for $48 \mathrm{~h}$ at $37^{\circ} \mathrm{C}$ with constant shaking. The water was changed every $8 \mathrm{~h}$ during the leaching period. This procedure yielded a highly porous, thin membrane. Sections were cut from the resulting films $(1.3 \times 1.5 \mathrm{~cm})$, with a razor blade, and rolled around Teflon cylinders with an outer diameter of $0.32 \mathrm{~cm}$. The surfaces of the films that were adjacent to the aluminum backed tape were always placed adjacent to the Teflon cylinder. The overlapping ends of the film were sealed together by briefly exposing one edge to chloroform, and manually pressing the overlapping ends together. The chloroform temporarily dissolved the polymer on the surface of each of the overlapping ends, and after the chloroform evaporated the overlapping ends were sealed together. The tubes were then slipped off of the Teflon template. The ends were closed by placing a circular piece of the same porous films over the ends, and sealing as above with chloroform. Tubes $1.5 \mathrm{~cm}$ long, with an inner diameter of $0.32 \mathrm{~cm}$ resulted. Tubes were lyophilized to remove residual solvent, and sterilized by exposure to ethylene oxide for $24 \mathrm{~h}$ at room temperature. 


\section{POLYMERS OF LACTIC AND GLYCOLIC ACID}

\section{Device Characterization}

For scanning electron microscopic examination, samples were gold coated using a Sputter Coater (Desk II, Denton Vacuum, Cherry Hill, NJ). An environmental scanning electron microscope (ElectroScan; Wilmington, MA) was operated at $30 \mathrm{kV}$ with a water vapor environment of 5 Torr to image samples. Photomicrographs were taken with Polaroid 55 film.

The porosity and pore size distribution of devices was analyzed by mercury porosimetry (Poresizer 9320 , Micromeritics, Norcross, GA) using a solid penetrometer with a 5-ml bulb volume (920-61707-00, Micromeritics). The void volume and pore size distribution of polymer devices were determined as previously described. ${ }^{10}$

Thermal mechanical analysis was performed with a TMA 7 (Perkin Elmer Corp; Norwalk, CT) using a compression probe with a circular tip $(d=3.0 \mathrm{~mm})$. All testing was done at a constant temperature of $37^{\circ} \mathrm{C}$. Tubes were placed on their sides for testing (axis of tube lumen perpendicular to axis of force application), and the change in device diameter (parallel to direction of force application) was followed during and after force application. The compressional forces applied to the tubes in vivo will also be exerted predominantly in a radial direction. The resulting deformations were normalized to the initial device diameter.

The degradation and erosion characteristics of bonded devices was assayed by placing individual tubes

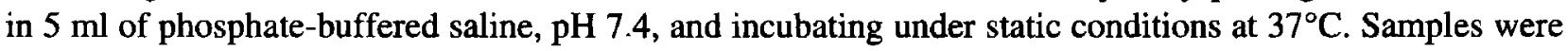
removed from this solution at set times, air dried, lyophilized, and weighed. The degradation of devices was determined by dissolving samples in chloroform and determining the molecular weight using gel permeation chromatography. The mass loss was analyzed by weighing lyophilized devices before and after the incubation period. The release of lactic acid was assayed enzymatically with lactic dehydrogenase using a kit from Sigma Chemical. The release of glycolic acid was quantitated with a colorimetric assay, ${ }^{11}$ which involves decarboxylating glycolic acid in the presence of concentrated sulfuric acid to form formaldehyde, followed by reaction of formaldehyde with chromotropic acid to yield a colored product that can be quantitated spectrophotometrically.

\section{Implantation of Devices}

Polymer constructs were implanted into the mesentery and omentum of Lewis rats as previously described. ${ }^{9}$ The mesenteric tissue or omentum was rolled around the devices to promote tissue invasion and neovascularization of the implants from all sides. Implants were secured in place with sutures of 7-0 Ethilar (Davis and Geck). The implants were subsequently removed after 7-28 days, and fixed in 10\% buffered formalin. NIH guidelines for the care and use of laboratory animals (NIH Publication 85-23 Rev. 1985) have been observed in all experiments involving animals. Inhalation anesthesia with methoxyflurane was always utilized. Thin sections were cut from paraffin-embedded tissue, and histological sections were stained with hematoxylin and eosin. Photomicrographs were taken with Kodak Tmax film using a Nikon inverted microscope.

\section{RESULTS}

\section{Fabrication and in Vitro Characterization of Devices}

The pore structure of films formed with the particulate leaching technique could be controlled by varying the ratio of polymer to salt in the film fabrication process. Films fabricated with a polymer/salt ratio of $1 / 3$ exhibited large pores on the air surface, approximately the size of the salt particles utilized to form the pores (Fig. 1A). These films had much smaller pores on the surface of the film exposed to the aluminum backed tape-coated glass surface (Fig. 2A). Decreasing the ratio of polymer to salt from $1 / 3$ to $1 / 24$ resulted in the formation of larger pores on the air surface of the films (Fig. 1A-D), and these pores were not as uniform as the pores formed at lower salt loadings. Larger pores also formed on the film surface exposed to the glass dish as the polymer to salt ratio was decreased (Fig. 2A-C). At a very low ratio (1/12) both sides of the films exhibited a similar pore structure. Films fabricated from all polymers (PLLA, PDLLA, 


\section{MOONEY ET AL.}
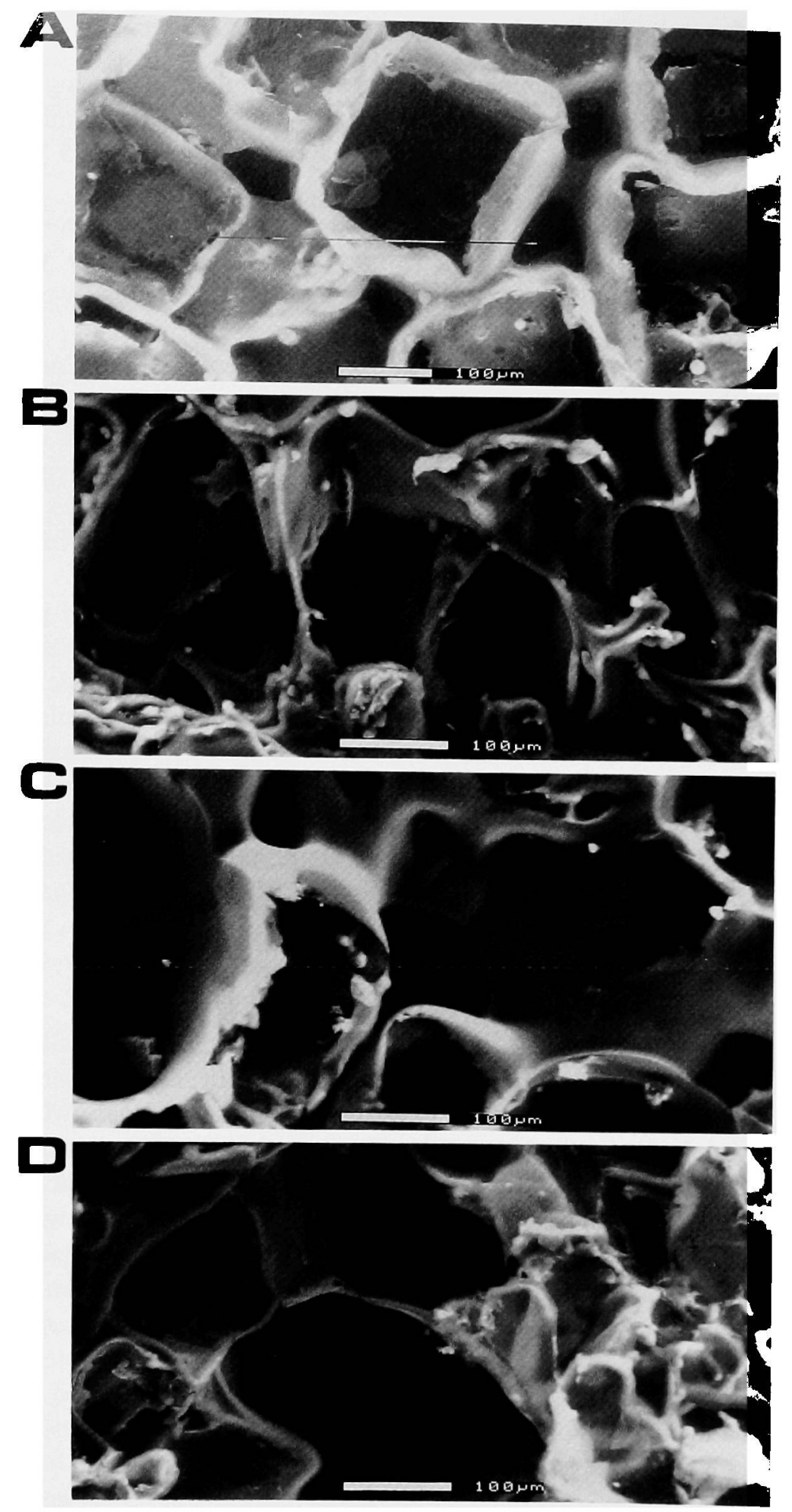

FIG. 1. The pore structure of polymer films on the air exposed surface was controlled by the ratio of polymer/salt. This ratio was varied from $1 / 3$ (A), 1/6 (B), 1/12 (C), to 1/24 (D). Size bars are present on the photomicrographs.

50/50 PLGA, and 85/15 PLGA) could be readily formed into hollow tubes (Fig. 3). There was no significant differences in the pliability of films formed from the different polymers. However, films formed using a polymer/salt ratio lower than 1/12 were quite brittle, and the manipulation needed to form tubes from these films often resulted in their fracture. Films fabricated using a polymer/salt ratio of $1 / 3$ were utilized to fabricate the tubular devices used in all subsequent studies. These films had a thickness of $320 \pm 50 \mu \mathrm{m}$ 
S E N D F F O R R Y O U R

C O M P L I M E N T A R Y

S U B S C R I P T I O N

\section{TO}

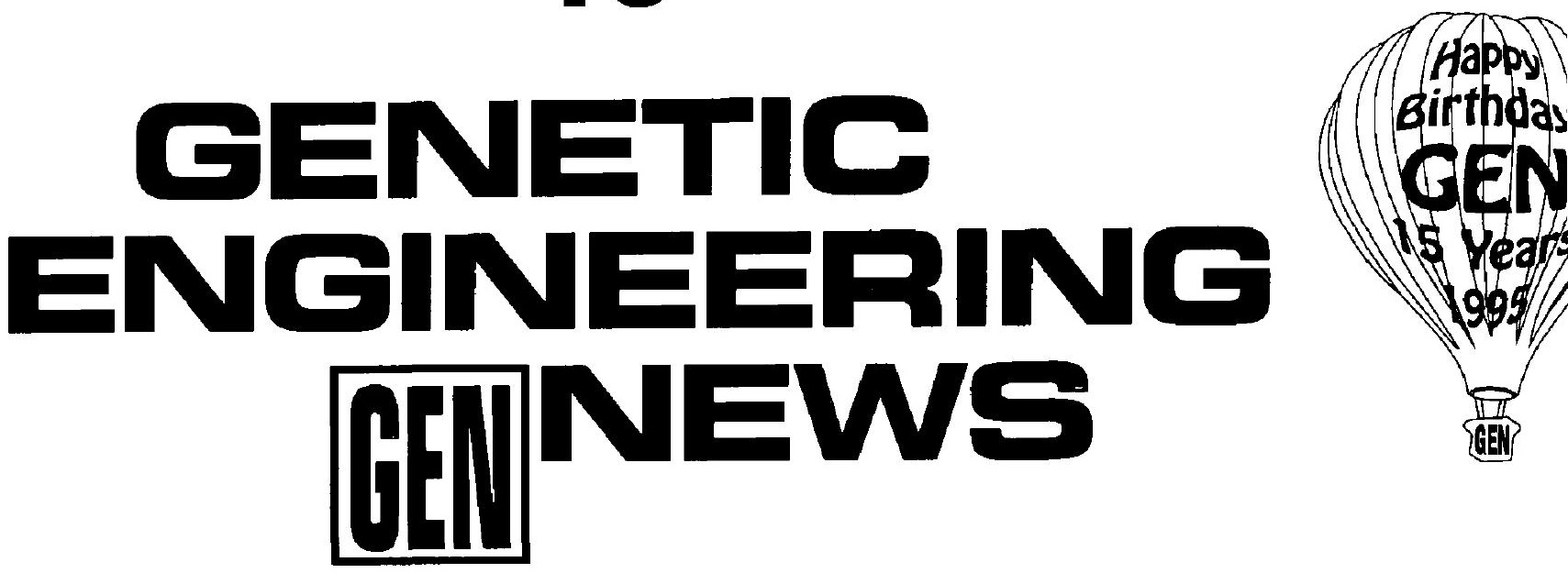

Mail this postage paid card TODAY!

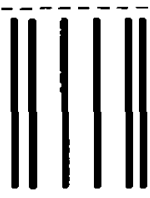

\section{NO POSTAGE NECESSARY \\ IF MAILED \\ IN THE \\ UNITED STATES}

\section{BUSINESS REPLY CARD FIRST CLASS PERMIT NO. 5443 NY, NY}

POSTAGE WILL BE PAID BY ADDRESSEE

GENetic ENGineERING NEWS

2 MADISON AVE.

LARCHMONT, NY 10538-9952 

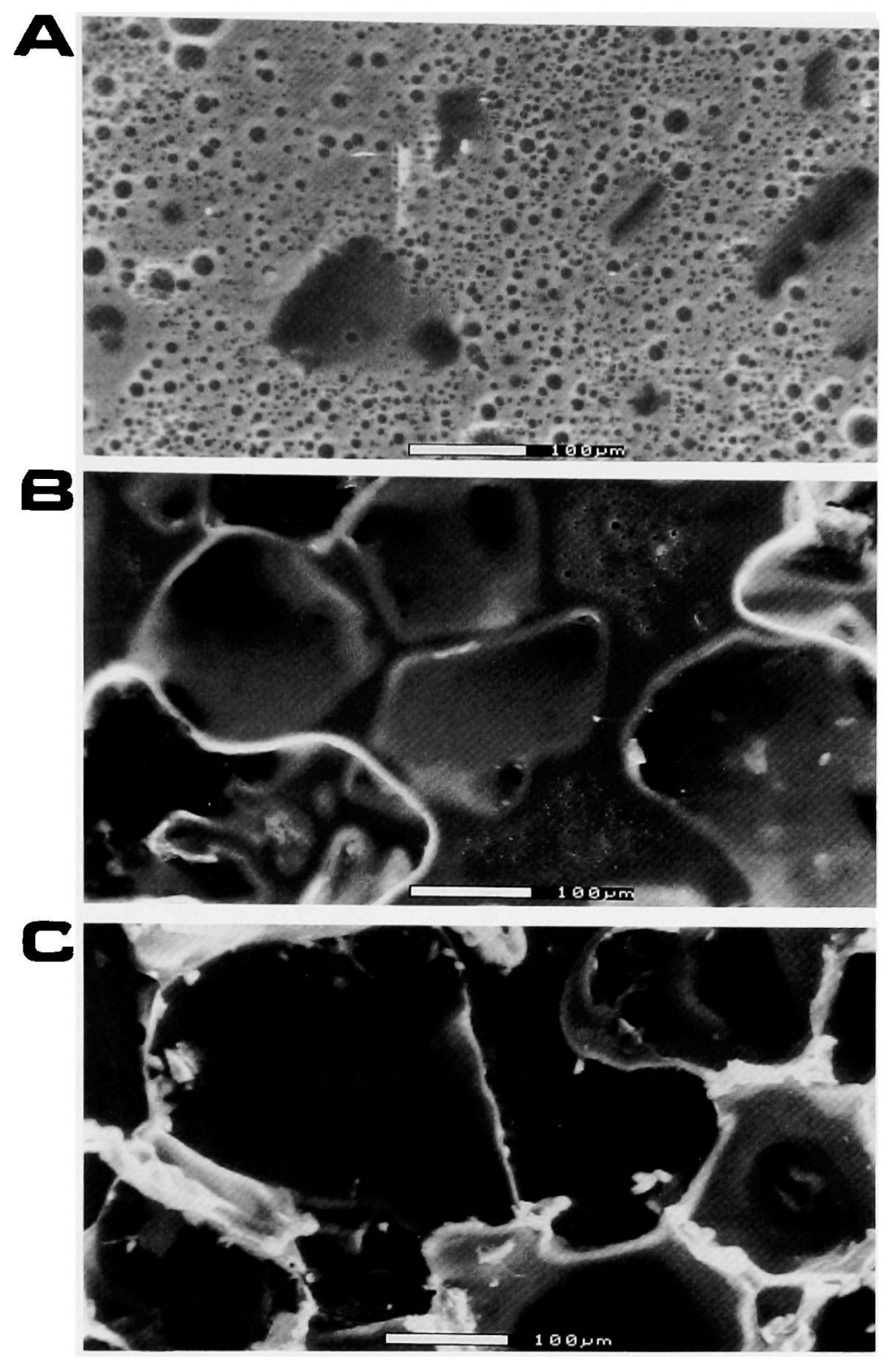

FIG. 2. The pore structure of the polymer films on the surface exposed to the glass petri dish depended on the ratio of polymer/salt utilized to fabricate the films. The ratio was varied from 1/3 (A), 1/6 (B), to $1 / 12$ (C). A size bar is shown on the photomicrograph.

(mean $\pm \mathrm{SD}, n=36)$, a porosity of $87 \pm 4 \%(n=12)$, and a volume average pore diameter of $150 \pm 50$ $\mu \mathrm{m}(n=12)$.

To determine the erosion characteristics of devices fabricated from various polymers, devices were placed in a phosphate-buffered saline solution and maintained at $37^{\circ} \mathrm{C}$ under static conditions for varying times. Devices fabricated from 50/50 PLGA began to lose significant mass after approximately 7 weeks, and were completely eroded (100\% mass loss) by 10 weeks (Fig. 4A). Devices fabricated from 85/15 PLGA did not exhibit any weight loss until after 10 weeks, but were completely eroded by 35 weeks. PDLLA devices exhibited no mass loss until 20 weeks, and PLLA devices had no weight loss over 40 weeks. No significant monomer release was found until the polymer had degraded to a low molecular weight (approximately 9000) (Fig. 4B). D-Lactic acid and L-lactic acid were released with identical kinetics from 50/50 devices, and glycolic acid was released more rapidly (Fig. 4B).

The compression resistance of devices was next tested. Devices fabricated from all four polymers resisted compression by moderate forces $(50 \mathrm{mN})$, but the ability of devices to withstand a larger compres- 


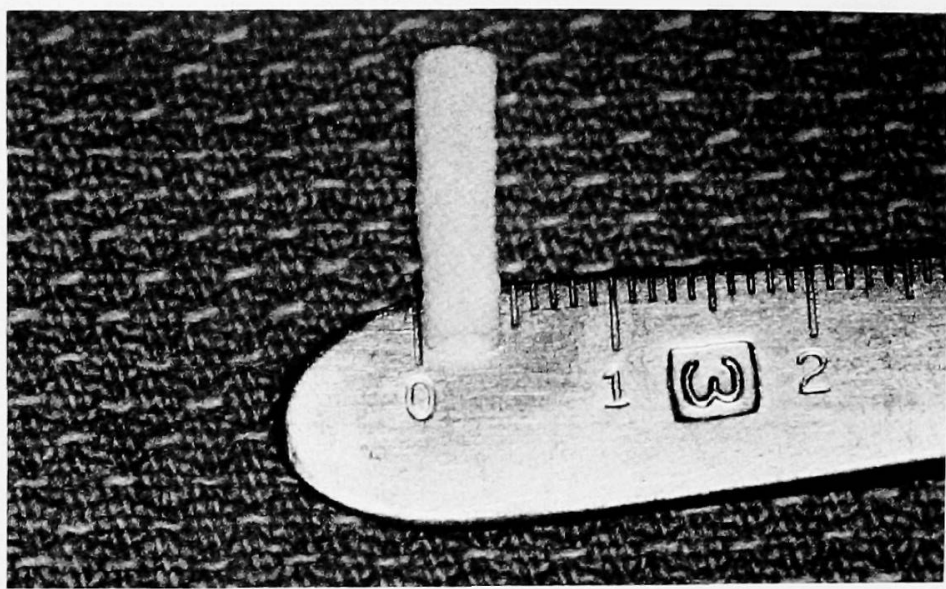

FIG. 3. A photograph of a tubular device fabricated from a porous film of 50/50 PLGA.

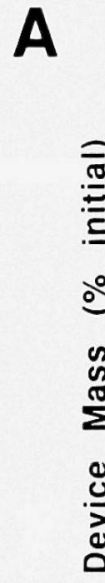

FIG. 4. In vitro erosion of tubular devices. (A) The erosion kinetics (mass loss) of devices was regulated by the polymer from which they were fabricated. All masses were normalized to the initial device mass. Values represent the mean and standard deviation calculated from 4 samples at each time point. (B) The molecular weight (MW: $\diamond)$ of devices fabricated from 50/50 PLGA rapidly decreased in vitro. Glycolic acid (ם), D-lactic acid ( $\mathbf{(})$, and Llactic acid $(\boldsymbol{\Delta})$ were released from the devices after the molecular weight reached a low value. Values for the monomer release were normalized to the monomer mass initially present in the device. The maximum monomer that could be released per device was calculated using the measured initial device mass. Values for the polymer molecular weight were normalized to the initial molecular weight.

B
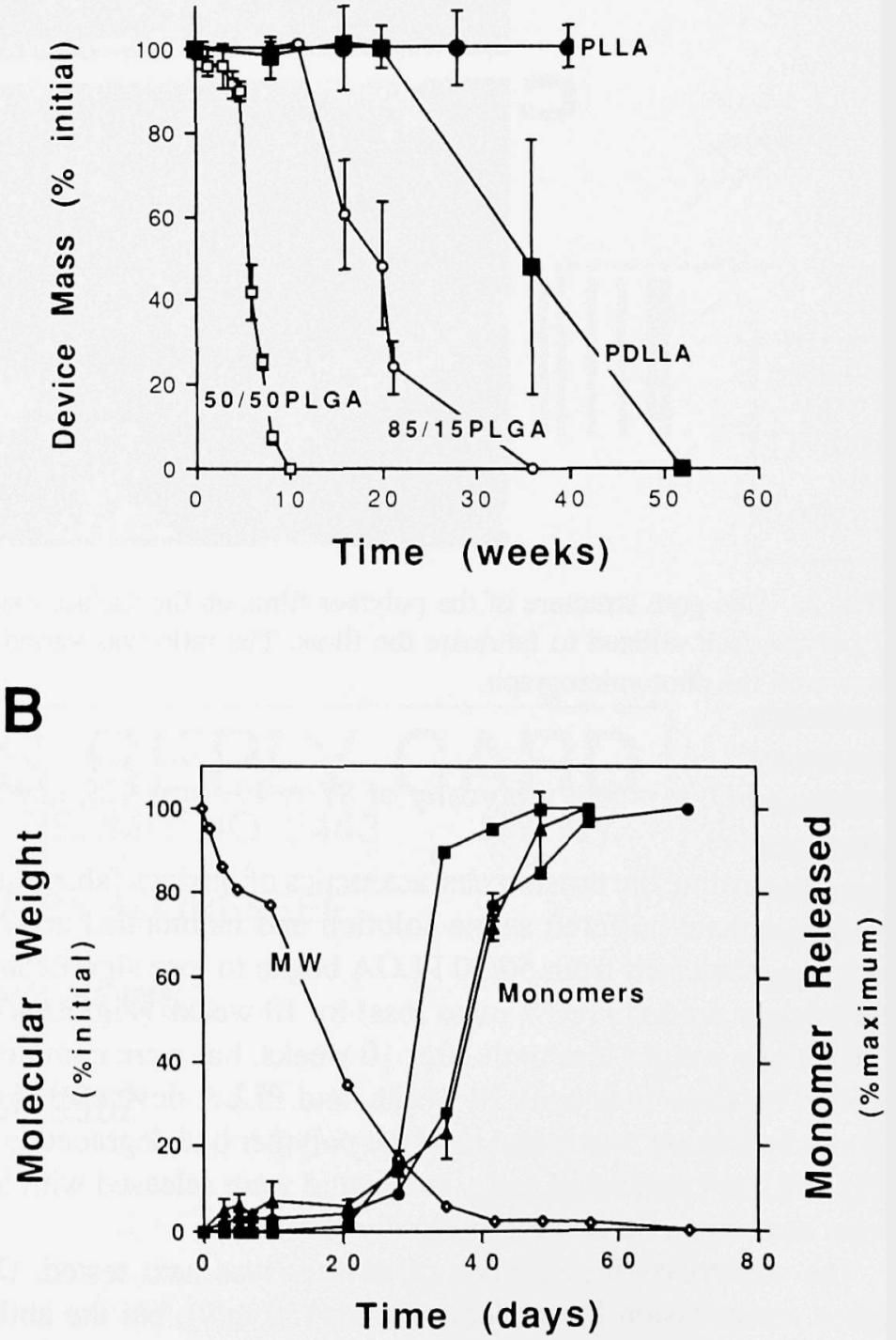

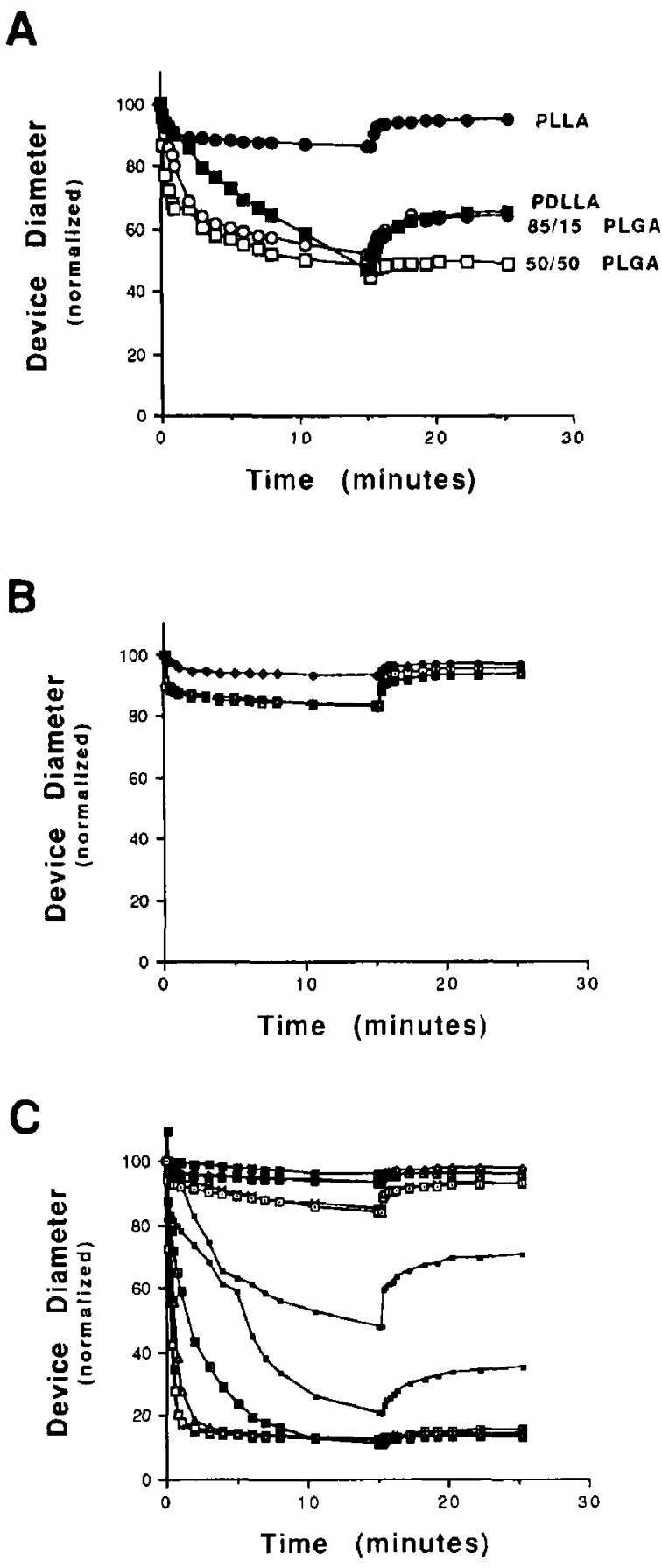

FIG. 5. Compression of tubular devices following the application of a compressive force. (A) Average strain responses of devices fabricated from different polymers that were subjected to a compressive force of $150 \mathrm{mN}$. The force was applied in a direction perpendicular to the axis of the device lumen starting at $0 \mathrm{~min}$. The force was removed at $15 \mathrm{~min}$, and the change in the diameter of the tube (parallel to the direction of force application) was monitored both during and after the time of force application. Values were normalized to the initial diameter. Strain values represent the mean calculated from 5 to 16 measurements with different tubes. (B) Individual strain diagrams of devices fabricated from PLLA. (C) Individual strain diagrams of multiple devices fabricated from 50/50 PLGA exhibit the inconsistant compression resistance of these devices at this loading.

sional force $(150 \mathrm{mN})$ was dependent on the polymer used in fabrication. PLLA tubes exhibiting the least compression under this force, and 50/50 PLGA devices exhibiting the greatest (Fig. 5A). The response to compressional forces was viscoelastic in all cases, as the devices only partially decompressed after the force was removed. While devices fabricated from PLLA and PDLLA consistantly resisted this load (Fig. 5B), 
PLGA devices did not exhibit a consistent response. The 50/50 PLGA tubes showed the greatest variability, as testing of multiple tubes gave widely varying results (Fig. 5C). Devices that compressed greater than $50 \%$ after force application typically showed little elastic recoil after the force was removed. Thus, devices that compressed more than this were considered to fail at this loading, and the failure rates of devices fabricated from the various polymers is given in Table 1.

\section{Implantation of Devices}

The devices fabricated with this technique will be utilized to transplant cells and serve as a template guiding the formation of a tubular tissue structure from the transplanted cells and the host tissue. Devices were implanted in the mesentery and omentum of Lewis rats to determine if they would guide the formation of a tubular tissue in vivo. These sites are easily accessed surgically, are well vascularized, and are attractive locations to engineer soft tissues. ${ }^{12}$ Devices were implanted by placing them on the omentum or on a leaf of the mesentery that had been isolated on surgical gauze (Fig. 6A), and then rolling the mesentery/omentum over the device (Fig. 6B). Devices were immobilized using several sutures, and the mesentery/omentum and construct were replaced within the abdominal cavity. In the first days following implantation, there was an acute inflammatory response to the devices. Fibrin deposition and macrophage infiltration were noted within the pores of the devices by 3 days (Fig. 7A). Fibrovascular tissue formed in the pores of the devices and on the luminal surface over 7-14 days (Fig. 7B). Capillary ingrowth through the pores accompanied the fibrous tissue ingrowth (Fig. 7C). Fibrovascular tissue occupied a greater percentage of the devices' lumens over time, until the lumen was completely filled in with this tissue by 28 days. The closure of the devices' lumens would presumably be prevented by a lining of epithelial cells (e.g., transplanted enterocytes). The ability of devices to maintain their structure and induce the formation of a tubular tissue was dependent on the polymer utilized to fabricate the devices. All PLLA and PDLLA devices maintained their structure in vivo, while devices fabricated from the other polymers were not as stable (Table 1).

\section{DISCUSSION}

Tubular devices have been fabricated from biodegradable polymers of lactic and glycolic acid using a simple, easily reproduced technique. The pore structure of the devices can be controlled by the processing conditions. The erosion kinetics and mechanical properties of the devices can be controlled by varying the polymer utilized to fabricate the devices. These devices can be used to engineer tubular tissue structures in laboratory animals.

The porosity of an implanted device affects the diffusion of factors into the device, the mechanical prop-

Table 1. In Vitro and In Vivo Compression Test Failure of Devices

\begin{tabular}{lccccc}
\hline & \multicolumn{2}{c}{ In vitro } & & \multicolumn{2}{c}{ In vivo } \\
\cline { 2 - 3 } \cline { 5 - 6 } Polymer & $\begin{array}{c}\text { Number } \\
\text { samples }\end{array}$ & $\begin{array}{c}\text { Failure } \\
\text { rate }\end{array}$ & & $\begin{array}{c}\text { Number of } \\
\text { samples }\end{array}$ & $\begin{array}{c}\text { Failure } \\
\text { rate }\end{array}$ \\
\hline PLLA & 6 & $0 \%$ & 2 & $0 \%$ \\
PDLLA & 5 & $20 \%$ & 6 & $0 \%$ \\
85/15PLGA & 5 & $40 \%$ & 9 & $54 \%$ \\
50/50PLGA & 16 & $58 \%$ & 6 & $50 \%$ \\
\hline
\end{tabular}

${ }^{a}$ Compression testing was performed with a force of $150 \mathrm{mN}$. A device was considered to fail if it compressed to less than $50 \%$ of the original diameter.

${ }^{b}$ Devices were implanted into the mesentery or omentum of Lewis rats for 7-28 days. Histological sections were examined, and devices were considered to fail if they compressed and did not maintain their original, tubular shape. 


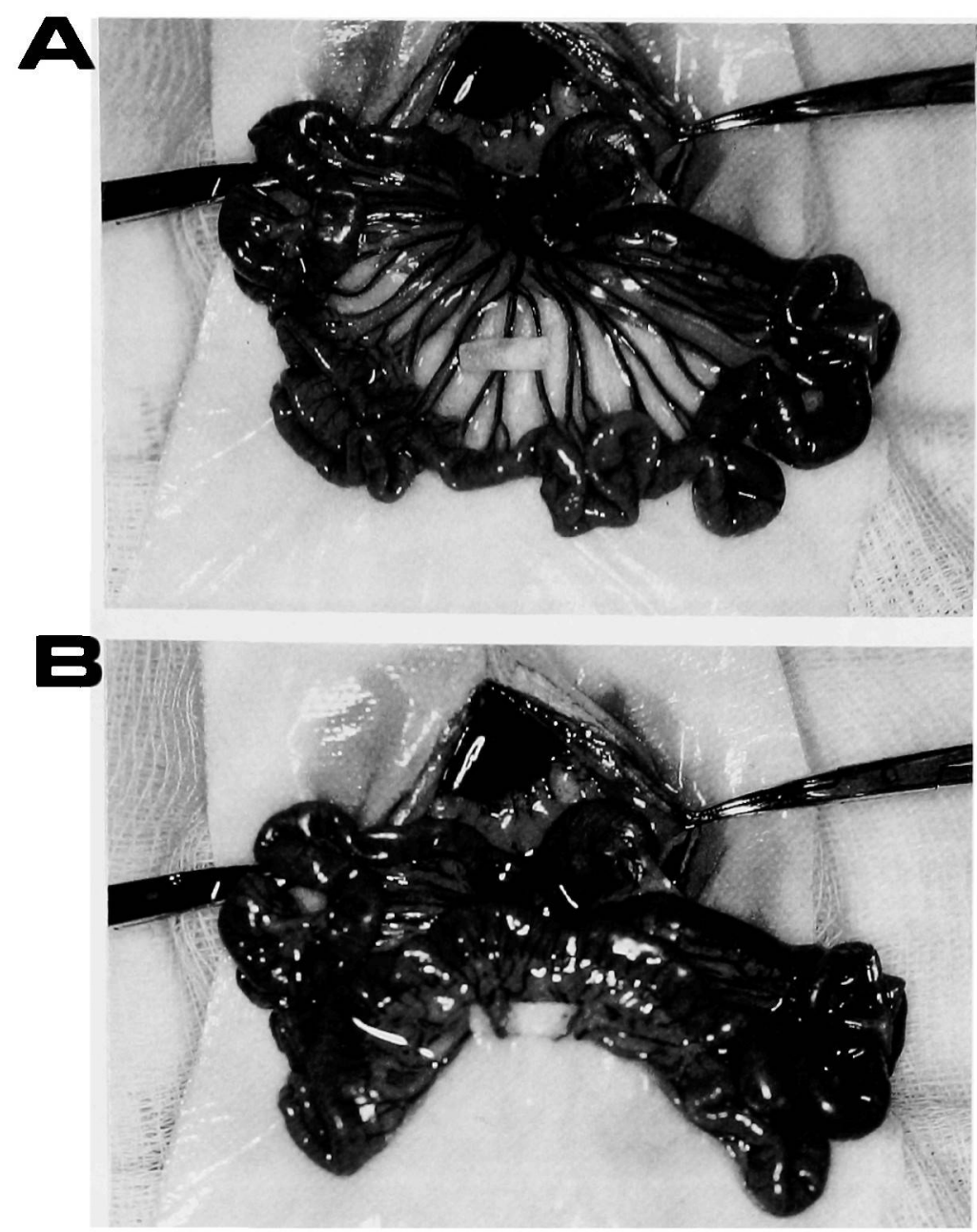

FIG. 6. Photographs of a tubular device being implanted in the mesentery of a Lewis rat. The device was placed on the mesentery (A), and the mesentery was subsequently wrapped around the device (B) and sutured in place before implantation.

erties, and the ability of fibrovascular tissue to invade the device. Fibrovascular tissue ingrowth is critical if one desires to engineer a tissue that is structurally integrated with the host tissue. This process leads to the formation of a vascular network that supplies the metabolic needs of the developing tissue. The rate and pattern of tissue ingrowth are functions of the devices' pore structure and size. ${ }^{13-17}$ The pore structure of devices fabricated in this study was not symmetrical unless a very low ratio of polymer/salt was utilized in the fabrication procedure. The small pores on the luminal surface of the devices may be advantagous for some applications as they allow cells seeded onto the luminal surface to readily organize into a confluent sheet. ${ }^{9}$ However, this asymmetry likely slows diffusion of factors between the luminal side and exterior of the device, and may also retard fibrovascular tissue ingrowth.

Different erosion times may be required of devices utilized to engineer various tissues (e.g., blood vessels versus intestine). The erosion kinetics of devices fabricated in this study were governed by the polymer utilized to fabricate the devices. The time for complete erosion could be varied between 10 weeks and over a year simply by varying which polymer was utilized to fabricate the device. The timing of device erosion is in general agreement with past studies utilizing these polymers. ${ }^{4,18,19}$ The thin membranes of these devices experience bulk degradation, and little mass loss or monomer release is noted until the polymer molecular weight has fallen quite low (Fig. 4). This leads to a relatively rapid monomer release, which could potentially acidify the tissue surrounding the devices. However, the low mass of monomer in highly porous devices such as these is unlikely to lead to any significant systemic problems. Also, the diffusional 


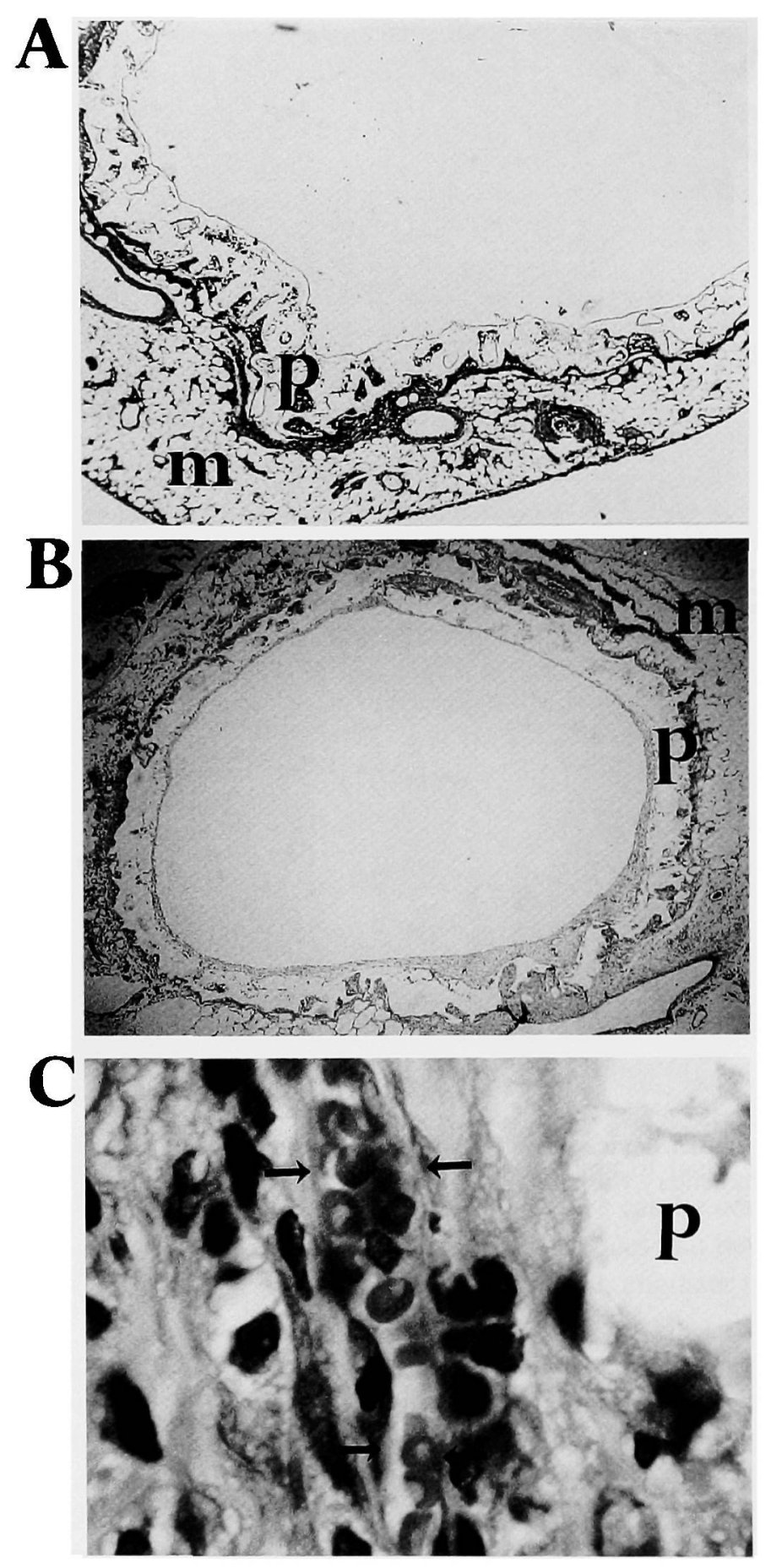

FIG. 7. Photomicrographs from histological sections of implanted 85/15 PLGA devices. (A) Devices implanted for 3 days exhibited fibrin deposition and macrophage infiltration of the devices' pores. (B) Fibrovascular tissue formed in the pores and luminal surface of implanted devices by 8 days. (C) A capillary (arrows) that formed in a pore of a device. This capillary contains numerous red blood cells. The mesenteric tissue $(\mathrm{m})$ surrounding the implants was large comprised of fat cells. The polymer ( $p$ ) does not prick either hematoxylin or eosin stain, and appears clear in these photos

and convective transport of fluids through the tissue will likely clear the monomers rapidly with little local build-up.

The compression resistance of devices utilized in tissue engineering applications is a critical feature. If the devices are to serve as templates, guiding the development of the desired tissue structure, they must maintain their own structure. The compression resistances of the tubular devices fabricated in this study 
were dependent on the polymer utilized to fabricate the devices. Devices fabricated from both PLLA and PDLLA resisted compressional forces and maintained their structure both in vitro and in vivo. Devices fabricated from PLGA did not resist compressional forces as well either in vitro or in vivo. The compression resistance of tubes fabricated from 50/50 PLGA was inconsistant at high compression forces (150 mN). While the magnitudes of the compressive forces that are exerted on implanted devices by the surrounding tissue are unclear, they are significant and will likely vary depending on the implant site. Devices that were stable to large compressional forces in vitro $(150 \mathrm{mN})$ were also stable after implantation into the mesentery of laboratory rats. The results of the current study suggest that devices fabricated from PLGA may need to be mechanically stabilized if they are to maintain their structure in vivo.

The compression resistances of devices were studied in vitro by applying a constant force on the tubes. Contact between the compression tip and the tubes was not analyzed, and will likely change as the tubes compress. For this reason, results were reported for compressional forces, not stresses. Calculation of stresses using the entire contact area of the compression probe would give the most conservative estimate of mechanical moduli.

In summary, the tubular devices described in this report may be useful for transplanting a variety of cell types to engineer different tissues. Cells can be readily seeded into the devices, where they adhere and organize. ${ }^{9}$ A tubular, vascularized tissue is created by implanting these devices in experimental animals. In the present study, fibrovascular tissue ingrowth continued until the lumen of the devices was filled, but an epithelial cell lining (e.g., transplanted enterocytes) would likely prevent this closure. The erosion time and mechanical properties of these devices can be predicted, and matched to the requirements for a specific time.

\section{ACKNOWLEDGMENTS}

The authors acknowledge the valuable assistance provided by Dr. Betsy Schloo, who prepared all histological sections. Financial support for this work was provided by the National Science Foundation (BCS9202311) and Advanced Tissue Sciences.

\section{REFERENCES}

1. Langer, R., and Vacanti, J.P. Tissue engineering. Science 260, 920, 1993.

2. Mooney, D.J., and Vacanti, J.P. Tissue engineering using cells and synthetic polymers. Transplant. Rev. 7, 153, 1993.

3. Frazza, E.J., and Schmitt, E.E. A new absorbable suture. J. Biomed. Mater. Res. 1, 43, 1971.

4. Gilding, D.K. Biodegradable polymers. In Williams, D.F. ed. Biocompatibility of Clinical Implant Materials. Boca Raton, FL: CRC Press, 209, 1981.

5. Organ, G.M., Mooney, D.J., Hansen, L.K., Schloo, B., and Vacanti, J.P. Transplantation of enterocytes utilizing polymer-cell constructs to produce neointestine. Transplant. Proc. 24, 3008, 1992.

6. Weinberg, C.B., and Bell, E. A blood vessel model constructed from collagen and cultured vascular cells. $\underline{\text { Science }}$ 231, 397, 1986.

7. Sakata, J., Vacanti, C.A., Schloo, B., Healy, G.B., Langer, R., and Vacanti, J.P. Tracheal composites tissue engineered from chondrocytes, tracheal epithelial cells, and synthetic degradable scaffolds. Transplant. Proc. 26, 3309, 1994.

8. Atala, A., Freeman, M.R., Vacanti, J.P., Shepard, J., and Retik, A.B. Implantation in vivo and retrieval of artificial structures consisting of rabbit and human urothelium and human bladder muscle. J. Urol. 150, 608, 1993.

9. Mooney, D.J., Organ, G., Vacanti, J.P., and Langer, R. Design and fabrication of cell delivery devices to engineer tubular tissues. Cell Transplant. 3, 203, 1994.

10. Mikos, A.G., Thorsen, A.J., Czerwonka, L.A., Bao, Y., and Langer, R. Preparation and characterization of poly (Llactic acid) foams. Polymer 35, 1068, 1994.

11. Tan, S. A colorometric assay for glycolic acid. Clin. Chim. Acta 89, 13, 1978.

12. Johnson, L.B., Aiken, J., Mooney, D., Schloo, B., Cima, L., Langer, R., and Vacanti, J.P. The mesentery as a laminated vascular bed for hepatocyte transplantation. Cell Transplant. 3, 273, 1994 


\section{MOONEY ET AL.}

13. Wesloski, S.A., Fries, C.C., Karlson, K.E., Bakey, M., and Sawyer, P.N. Porosity: Primary determinant of ultimate fate of synthetic vascular grafts. Surgery 50,91, 1961.

14. White, R.A., Hirose, F.M., Sproat, R.W., Lawrence, R.S., and Nelson, R.J. Histopathologic observations after shortterm implantation of porous elastomers in dogs. Biomaterials 2, 171, 1981.

15. Mooney, D.J., Kaufmann, P.M., Sano, K., McNamara, K.M., Vacanti, J.P., and Langer, R. Transplanting hepatocytes on porous, biodegradable sponges. Transplant. Proc. 26(6), 4025, 1994.

16. Mikos, A.G., Sarakinos, G., Lyman, M.D., Ingber, D.E., Vacanti, J.P., and Langer, R. Prevascularization of porous biodegradable polymers. Biotech. Bioeng. 42, 716-723, 1993.

17. Wintermantel, E., Cima, L., Schloo, B., and Langer, R. Angiopolarity of cell carriers: Directional angiogenesis in resorbable liver cell transplantation devices. In Steiner, R., Weisz, P.B., and Langer, R., eds. Angiogenesis: Key Principles-Science-Technology-Medicine. Basel, Switzerland: Birkhauser Verlag, 331, 1992.

18. Holland, S.J., Tighe, B.J., and Gould, P.L. Polymers for biodegradable medical devices I. The potential of polyesters as controlled macrolmolecular release systems. J. Controlled Release 4, 155, 1986.

19. Schakenraad, J.M., Nieuwenhuis, P., Molenaar, I., Helder, J., Dijkstra, P.J., and Feijen, J. In vivo and in vitro degradation of glycine/DL-lactic acid copolymers. J. Biomed. Mat. Res. 23, 1271, 1989.

Address reprint requests to:

Robert Langer

E 25-342

MIT

77 Massachusetts Avenue

Cambridge, MA 02139 


\section{This article has been cited by:}

1. Sophia W. Liao, Xiao Lu, Andrew J. Putnam, Ghassan S. Kassab . 2007. A Novel Time-Varying Poly Lactic-Co Glycolic Acid External Sheath for Vein Grafts Designed under Physiological LoadingA Novel Time-Varying Poly Lactic-Co Glycolic Acid External Sheath for Vein Grafts Designed under Physiological Loading. Tissue Engineering 13:12, 2855-2862. [Abstract] [PDF] [PDF Plus]

2. Jun Pan, Yuanliang Wang, Suhua Qin, Bingbing Zhang, Yanfeng Luo. 2005. Grafting reaction of poly(D,L)lactic acid with maleic anhydride and hexanediamine to introduce more reactive groups in its bulk. Journal of Biomedical Materials Research Part B: Applied Biomaterials 74B:1, 476-480. [CrossRef]

3. N. Mao, S. J. Russell. 2004. Nonwoven Wound Dressings. Textile Progress 36:4, 1-57. [CrossRef]

4.F. Opitz, C. Melle, K. Schenke-Layland, I. Degenkolbe, D.P. Martin , F. von Eggeling, T. Wahlers , U.A. Stock . 2004. ProteinChip System Technology: A Powerful Tool to Analyze Expression Differences in Tissue-Engineered Blood VesselsProteinChip System Technology: A Powerful Tool to Analyze Expression Differences in Tissue-Engineered Blood Vessels. Tissue Engineering 10:3-4, 611-620. [Abstract] [PDF] [PDF Plus]

5. Robert Langer, Nicholas A. Peppas. 2004. Advances in biomaterials, drug delivery, and bionanotechnology. AIChE Journal 49:12, 2990-3006. [CrossRef]

6. Mona K. Marei, Samir R. Nouh, Mohamed M. Fata, Adham M. Faramawy . 2003. Fabrication of Polymer Root Form Scaffolds to Be Utilized for Alveolar Bone RegenerationFabrication of Polymer Root Form Scaffolds to Be Utilized for Alveolar Bone Regeneration. Tissue Engineering 9:4, 713-731. [Abstract] [PDF] [PDF Plus]

7. D.J. Aframian, R.S. Redman, S. Yamano, J. Nikolovski , E. Cukierman, K.M. Yamada, M.F. Kriete, W.D. Swaim, D.J. Mooney, B.J. Baum . 2002. Tissue Compatibility of Two Biodegradable Tubular Scaffolds Implanted Adjacent to Skin or Buccal Mucosa in MiceTissue Compatibility of Two Biodegradable Tubular Scaffolds Implanted Adjacent to Skin or Buccal Mucosa in Mice. Tissue Engineering 8:4, 649-659. [Abstract] [PDF] [PDF Plus]

8. Rosana Z. Domingues, Arthur E. Clark, Anthony B. Brennan. 2001. A sol-gel derived bioactive fibrous mesh. Journal of Biomedical Materials Research 55:4, 468-474. [CrossRef]

9. Kuen Yong Lee, David J. Mooney. 2001. Cell-interactive polymers for tissue engineering. Fibers and Polymers 2:2, 51-57. [CrossRef]

10. Ulrich A. Stock, Dmitri Wiederschain, Susan M. Kilroy, Dominique Shum-Tim, Philip N. Khalil, Joseph P. Vacanti, John E. Mayer, Marsha A. Moses. 2001. Dynamics of extracellular matrix production and turnover in tissue engineered cardiovascular structures. Journal of Cellular Biochemistry 81:2, 220-228. [CrossRef]

11. F Shen, Y?L Cui, L?F Yang, K?D Yao, X?H Dong, W?Y Jia, H?D Shi. 2001. A study on the fabrication of porous chitosan/gelatin network scaffold for tissue engineering. Polymer International 49:12, 1596-1599. [CrossRef]

12. Ralf Sodian, Jason S. Sperling, David P. Martin, Ana Egozy, Ulrich Stock, John E. Mayer Jr., Joseph P. Vacanti . 2000. Technical Report: Fabrication of a Trileaflet Heart Valve Scaffold from a Polyhydroxyalkanoate Biopolyester for Use in Tissue EngineeringTechnical Report: Fabrication of a Trileaflet Heart Valve Scaffold from a Polyhydroxyalkanoate Biopolyester for Use in Tissue Engineering. Tissue Engineering 6:2, 183-188. [Abstract] [PDF] [PDF Plus]

13. Byung-Soo Kim, David J. Mooney. 1998. Engineering smooth muscle tissue with a predefined structure. Journal of Biomedical Materials Research 41:2, 322-332. [CrossRef]

14. KAMAL H. BOUHADIR, DAVID J. MOONEY. 1998. In vitro and In vivo Models for the Reconstruction of Intercellular Signalinga,. Annals of the New York Academy of Sciences 842:1 SALIVARY GLAN, 188-194. [CrossRef]

15. Andrew J. Putnam, David J. Mooney. 1996. Tissue engineering using synthetic extracellular matrices. Nature Medicine 2:7, 824-826. [CrossRef] 